\title{
MQTT_SN_QoS: an enhanced MQTT protocol for wireless sensor network in smart parking
}

\author{
Hicham Ouldzira $^{1}$, Ahmed Mouhsen ${ }^{2}$, Mohamed Hassoun $^{3}$, Rachid Habachi ${ }^{4}$, Mustafa Elalami $^{5}$ \\ ${ }^{1}$ Laboratory of Engineering, Industrial Management and Innovation, \\ Departement of Electrical Engineering, Sciences and technology Faculty, Hassan $1^{\text {st }}$ University Settat, Morocco, \\ odzira@yahoo.fr \\ ${ }^{2}$ Laboratory of Engineering, Industrial Management and Innovation, \\ Departement of Electrical Engineering, Sciences and technology Faculty, Hassan $1^{\text {st }}$ University Settat, Morocco, \\ ahmed.mouhsen@uhp.ac.ma \\ ${ }^{3}$ Laboratory of Engineering, Industrial Management and Innovation, \\ Departement of Electrical Engineering, Sciences and technology Faculty, Hassan $1^{\text {st }}$ University Settat, Morocco, \\ hassoun.mohamed100@gmail.com \\ ${ }^{4}$ Laboratory of Engineering, Industrial Management and Innovation, \\ Departement of Electrical Engineering, Sciences and technology Faculty, Hassan $1^{\text {st }}$ University Settat, Morocco, \\ r.habachi@uhp.ac.ma \\ ${ }^{5}$ Laboratory of Engineering, Industrial Management and Innovation, \\ Departement of Electrical Engineering, Sciences and technology Faculty, Hassan $1^{\text {st }}$ University Settat, Morocco, \\ mustafa.elalami@gmail.com
}

\begin{abstract}
In recent years, wireless sensor networks (WSN) have attracted increasing interest from academia and industry. WSNs can be deployed in various types of environmental monitoring and information gathering applications. In this chapter, we present wireless sensor network architecture for vehicle parking management. In a parking management system, a number of sensor nodes are deployed in a parking field, indicating whether or not the parking spaces are occupied. These sensor nodes are equipped with a battery (cell) that provides the necessary power for their operation. This energy resource is very important for the sensor nodes and has a direct influence on the lifetime of the sensor nodes and thus of the entire WSN.

With the integration of the Internet of Things (IoT), we will design a system to offer remote parking reservation, to help drivers to easily locate their parking space, and to manage the lifetime of this WSN. We propose the MQTT_SN_QoS (Message Queuing Telemetry Transport for Sensors Network by Quality of Service) communication protocol with the integration of its QoS levels to overcome these problems, and we simulate the results on Matlab.
\end{abstract}

Key words :WSN, MQTT, MQTT_SN, QoS levels, Smart parking, IoT.

\section{INTRODUCTION}

Intelligent parking can be seen as a demand for IoT[1]. The Smart City concept aims to meet the needs of the population, to allow a more efficient use of general resources and more particularly to facilitate the daily life of citizens. In this study, we propose a new approach for intelligent parking systems via a WSN[2]. This solution is divided into three parts, namely a hardware part that consists of recognizing the status of the parking spaces and then sending this data to a server where it is stored, a software part in the form of a web application that allows interaction with users (drivers) in order to know the status of the spaces in order to make a reservation and a last part that is responsible for managing the energy consumption of our WSN.

Numbers of people buying a vehicle are increasing and that was one of many factors of traffic cause nowadays alongside with car parking problem contributing as major problem worldwide scale[3].

The objective of this system is to develop, integrate and test a new algorithmic approach to minimizing the energy consumption of the WSN and also to assist vehicle parking in urban areas. We show that it is possible to propose an efficient method that determines a route to find an available parking space that minimizes time expectancy, fuel consumption and pollution rates, all thanks to an energy-efficient WSN[4].

The originality and innovative aspect of our approach is based on two principles. The first principle is based on the management of the electrical energy of the wireless sensor nodes, which provides us with the status of available parking 
spaces in real time. For the latter, we have therefore proposed a communication protocol that is efficient in terms of energy and faithful in terms of information transmission. We evaluated the performance of our simulation approach on Matlab. The second principle concerns guidance as a resource: it is not a question of guiding towards a free space but of proposing a route that optimises the time it takes to find such a space. For that we determine in a zone centered on a given destination, the route to be taken by a vehicle to minimize its time expectancy to find a parking space as quickly as possible.

In this system, we offer a complete publication and subscription service to inform drivers of the parking spaces that correspond to their personal interests. The "Publish-Subscribe" messaging system avoids repeated searches for parking spaces, as drivers continue to receive up-to-date information while driving. We then showed that driver satisfaction can be affected by different modes of communication depending on their mobility.

We also start by presenting a state of the art of smart car parks that manage energy consumption in their WSN, and then we present our Smart Parking System for Congestion Avoidance (SPSCA).

\section{RELATED WORK}

Due to the growth in the number of vehicles on the roads and the poor management of the various car parks, there will always be traffic jams and parking search conflicts, it is essential to implement intelligent car parks in order to make it easier and safer for drivers to park. The strong points of intelligent parking are: ease of parking, organisation of traffic on the roads, reduction of air pollution, etc. The management of a car park via an WSN has been the subject of several research studies.

In [5], the authors carried out a study on the technologies used for parking management. The common objective of all car parks is to make it easier for motorists to find free spaces and to orient them within the car park. Information on the availability of parking spaces must be accessible to motorists so that they do not waste time looking for a free space and this contributes to the preservation of the environment. A parking management system should meet the following requirements:

The system shall provide all information and guidelines to help drivers find available parking spaces.

The system should ensure effective management of parking spaces (free and occupied) in order to maximiseutilisation and profitability.

The system should provide powerful functions to enable administrators to more easily manage cars in the car park.

In [6], the parking management system is divided into two subsystems. A first vehicle detection sub-system (VDS) and a second vehicle management system (VMS). The VDS detects the status of the parking spaces and sends the collected information to the VMS subsystem to provide it to the drivers. Another intelligent parking management system is proposed in [7]. The authors of [8] compare the use of different types of sensors (acoustic, light and magnetic) for parking management. The information from the different types of sensors is sent to a central server in a limited time. In this paper, we propose a parking management architecture in which we evaluate the energy consumption of the sensor nodes deployed in the car park, which is neglected in the proposed parking management architectures.

Mainetti et al., n.d. using a RFID to detect available space for parking then transmit it through IEEE 802.15.4 Network to Smart Gateway so the data can be forward to central server via GPRS [9] a research aims to reduce congestion rates in urban areas because of the large number of vehiclesseeking parking. Karaliopoulos [10] proposed a game theory model to see if drivers choose to compete with these limited street parking spaces. The driver's decision leads to three outcomes: drivers who go directly to the private parking lot, those who decide to compete on the street parking lot and find one, and those who finally fail to find a street parking lot and switch to the private parking lot.

Kokolaki [11] studied the same problem by considering a parking assistance system, which modifies the driver's decision according to the number of competitive vehicles and the number of parking spaces currently available. As a result, the competitor's amount varies over time thanks to the real-time information of the intelligent parking service.

Rajabioun et al [12] revealed the problem of information dissemination in the on-street parking guidance system. That is, real-time parking availability data is only useful when the driver is very close to the parking space.

Information on the location and availability of a parking space close to the destination is provided to drivers by the current GPS-based vehicle navigation system. Information on the current status of the parking space is provided. Therefore, they cannot guarantee a parking space when the driver reaches the facility. A scientific solution based on the use of past and current parking status is proposed in [13]. The Poisson process is used to model the availability of a parking space. An intelligent algorithm that helps the driver to choose the slot with the highest probability of being vacant is also presented in [13].

Faheem et al [14] conducted a one-year study of street parking, which focused on various issues related to street parking, including policy, planning, management and operations. Different methods and different cities were used to demonstrate these issues. The study also highlighted different challenges related to on-street parking, such as peer-to-peer exchange and the storage of parking information.

Arnott and Rowse [15] modelled parking as a function of spatial structure, trip generation technology, parking and travel technology, and steady-state conditions. The problem with this modelling is that it does not account for traffic congestion.

Chon et al. [16] presented a location-based system called NAPA (Nearest Available Parking Lot Application) (Figure 3 ). This system helps to locate parking lots on campus or in areas such as airports, but does not provide information on the 
availability of parking spaces.

\section{Interpretation}

Based on the above, we propose a parking management architecture in which we evaluate the energy consumption of the sensor nodes deployed in the parking lot, which is neglected in the proposed state of the art parking management architectures.

The design of a parking facility based on an WSN offers high accuracy and meets the requirements mentioned above. The use of WSN for car park management offers customer comfort, robustness and management flexibility. Our motivation is therefore to ensure the management of car parks via a WSN.

\section{PROPOSED SYSTEM}

\subsection{System architecture}

Intelligent parking can be seen as a demand for IoT. The Smart City concept aims to meet the needs of the population, to allow a more efficient use of general resources and more particularly to facilitate the daily life of citizens. In this study, we propose a new approach for intelligent parking systems via a RCSF. This solution is divided into three parts, namely a hardware part that consists of recognizing the status of the spaces and then sending this data to a server where it will be stored, a software part in the form of a web application that allows interaction with users (drivers) in order to know the status of the spaces in order to make a reservation, and a last part that is responsible for managing the energy consumption of our RCSF. The general organization chart of the proposed system is shown in Figure 2.

\subsection{Principle of operation}

\subsubsection{Hardware part}

The hardware was chosen wisely for a good functioning with the means we have at our disposal to realize an intelligent parking equally functional in real time We chose the Arduino integrated development environment, an electronic and embedded computing development platform that provides several programmable electronic cards, for the realization of the application we used the Raspberry pi 3 card. We used the MQTT-SN protocol that implements the Publisher/Subscriber paradigm (Publication/Subscription). In what follows, we will detail the hardware design as shown in figure 1.

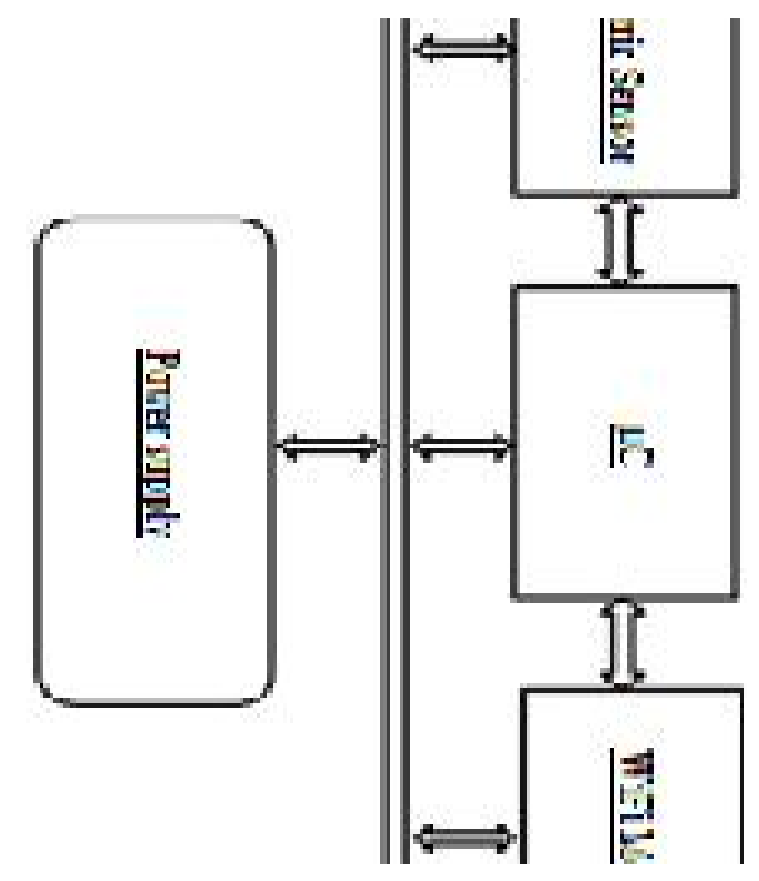

Figure 1: Hardware part of the SPSCA system

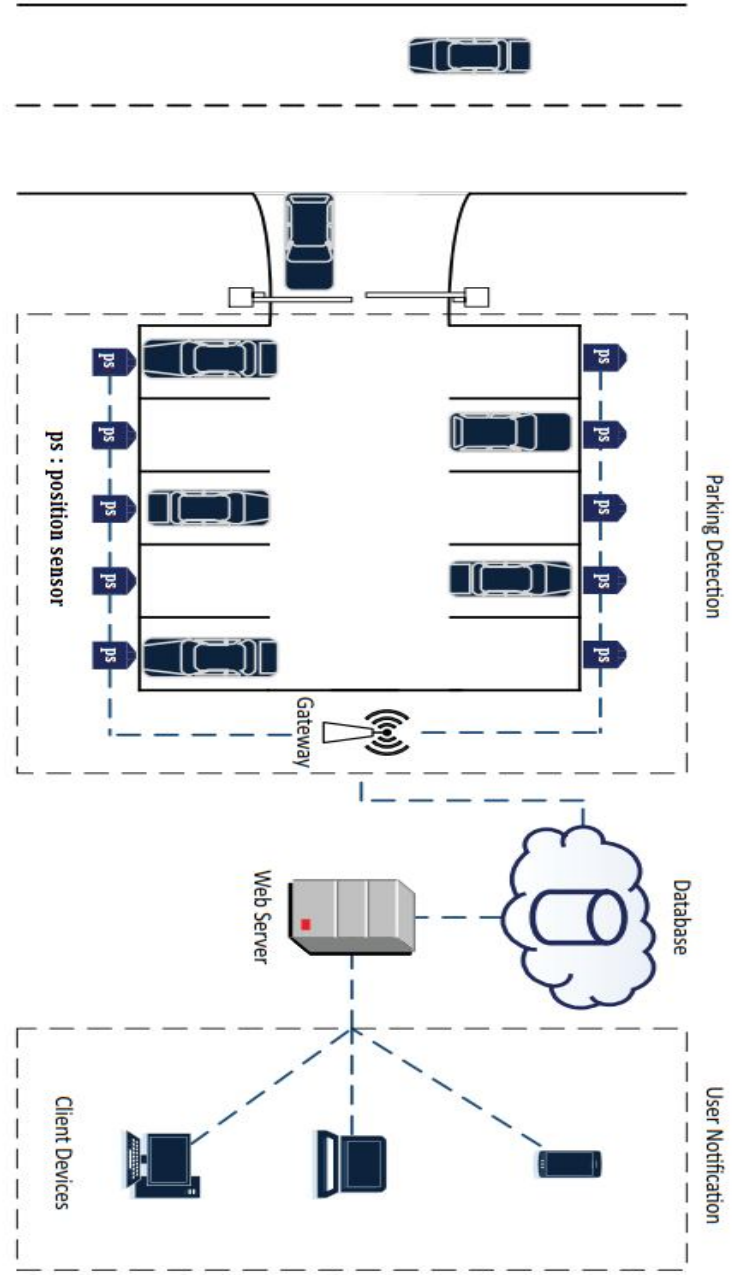

Figure 2: SPSCA System Architecture 


\subsubsection{Software part}

As we have already seen in section 3.2.1 (Hardware Architecture), we explain here the software design of the system.

First of all, we tested the status of the parking space in our smart car park at all times, using a wireless sensor that measures the distance between its position and the moving object. If this distance is more than $50 \mathrm{~cm}$, then there is a free space and the green LED lights up, but if this distance is less than $50 \mathrm{~cm}$, then there is an occupied space and the red LED lights up.

The flowchart in figure 3 describes the operation of the software of our 2SIEC.

If a slot $\mathrm{X}$ is reserved by a customer, the sensor of this slot is immediately put into standby mode, i.e. it does not deliver any information and the orange LED therefore lights up.

These wireless sensors are published their captured information on three Topics, namely energy, location and reservation of our MQTT-SN communication protocol, on the other hand the PW web page is only subscribed to the reservation and location Topics (figure 4).

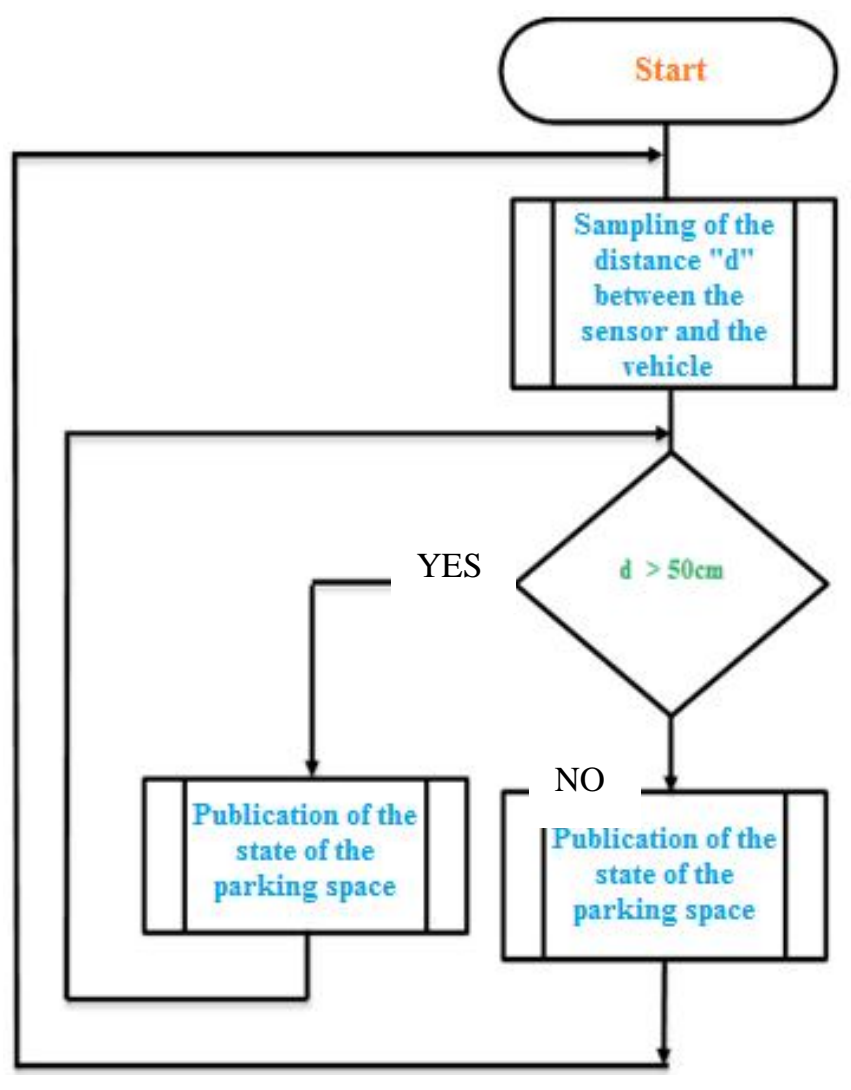

Figure 3: SPSCA System Architecture

\subsubsection{WSN}

Due to the computing power and battery constraints of the sensor nodes, WSNs often avoid sending information on the status of the system, which hinders the analysis of subsequent data. Thus, it can be seen that the inherent characteristics of intelligent parking pose problems in the power consumption of wireless sensor nodes that are not easily overcome with traditional solutions alone. This research work addresses this problem in the context of smart car parks, where WSNs have become an important data source but are known to be vulnerable at the same time.

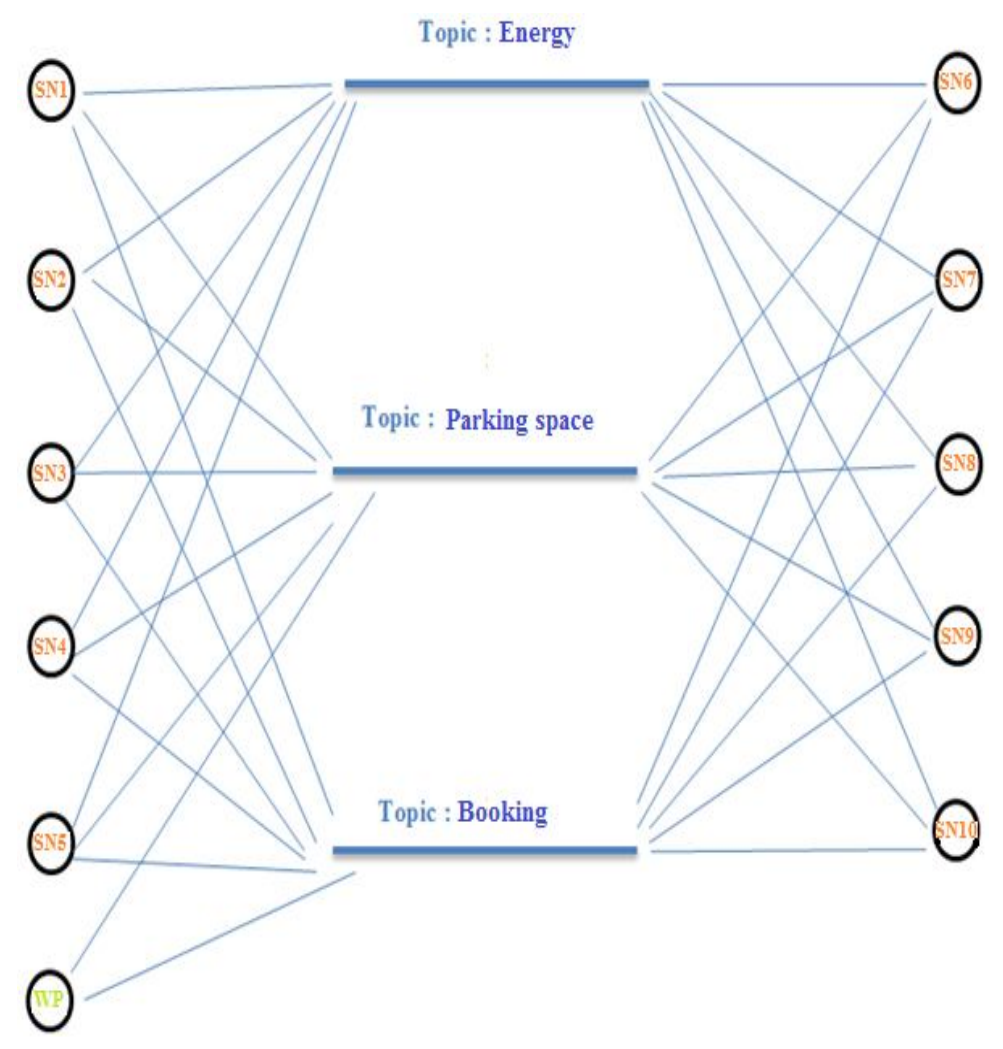

Figure 4:Publication and subscription of sensor nodes (SN) to Topics Energy, Parking space and Reservation

The MQTT protocol implements the publish/subscribe paradigm that provides three qualities of service (QoS) to ensure the exchange of messages between devices. However, the MQTT protocol is developed on the TCP stack and implements the TCP protocol to communicate. There is a version of MQTT for Sensor Networks (SN), called MQTT-SN, developed specifically for message exchange in wireless sensor networks (WSN). Since many intelligent devices will be connected on the same WSN, the network can be overloaded and the links can become unstable. This study presents a method to optimize message exchange, reduce network power consumption and increase message distribution during a communication process, between a publisher and a broker or between a publisher and middleware, by implementing the MQTT-SN protocol.

MQTT-SN is suitable for wireless sensor networks (WSN) and heterogeneous environments due to its small code footprint, low bandwidth usage and standardized interfaces. Finally, we evaluate this solution in terms of its QoS quality of service, which has three levels: 
QoS0: where the message will be delivered at most once.

QoS1: where the message will be delivered at least once.

QoS2: where the message will be obligatorily saved until it is received by the subscriber.

Figure 5 shows the architecture of the WSN used for our SPSCA System.

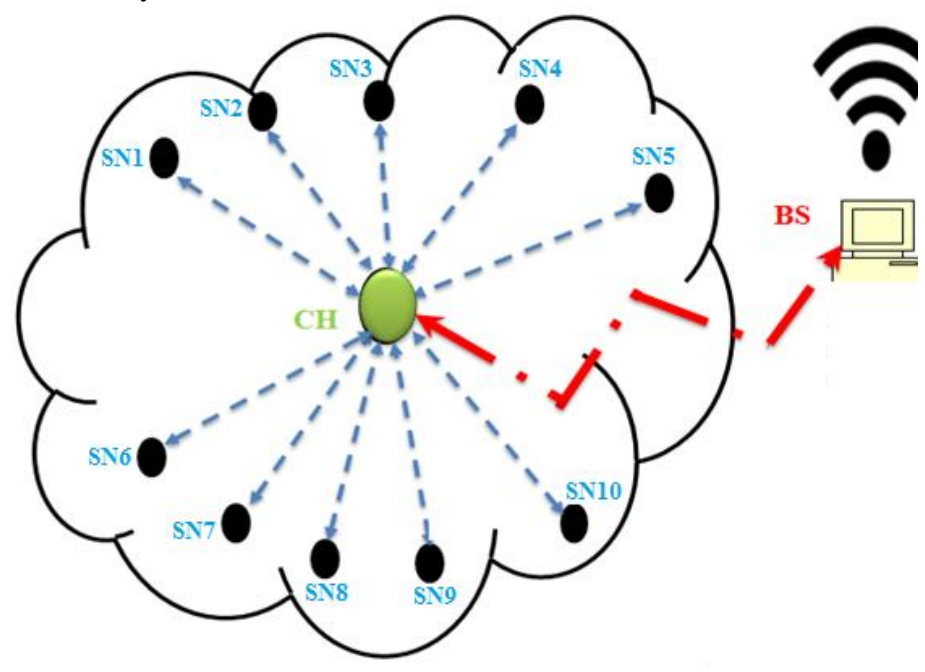

Figure 5:WSN architecture used

\subsection{IMPLEMENTATION}

In this section, we will present an intelligent parking for the parking of vehicles and remote reservation of the location via a web application. Next, we will present the demonstrative model of the intelligent parking.

Our realization (figure 6), describes the functioning of detection of the state of the parking space (occupied/free/reserved) within a car park. This detection is translated by the visualization of the three Green/Red/Orange LEDs. If the green LED of a parking space lights up, this means that the space is free, if the red LED of a parking space lights up, this means that the space is occupied, if the orange LED of a parking space lights up, this means that the space is reserved by a customer.

In the Figure 7 shows the access interfaces that interact with the user (driver) in order to find a free space.

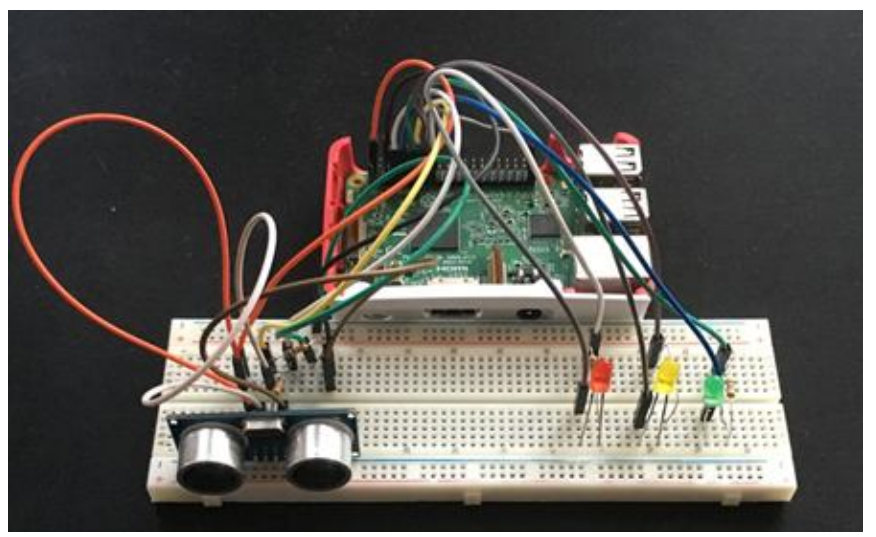

Figure 6: Realization prototype for a parking space

\subsection{SIMULATION OF RESULTSDISCUSSION}

Table 1: Simulation parameters

\begin{tabular}{|c|c|}
\hline Simulation area & $100 \times 100 \mathrm{~m}^{2}$ \\
\hline Number of nodes & 10 \\
\hline Packet size & 55 bytes \\
\hline Initial node energy & $2 \mathrm{j}$ \\
\hline Transmission rate & $250 \mathrm{kbs}-1$ \\
\hline Transmission power & $24.75 \mathrm{~mW}$ \\
\hline Receiving power & $13.5 \mathrm{~mW}$ \\
\hline Idle power & $6.5 \mathrm{~mW}$ \\
\hline Sleep power & $15 \mu \mathrm{W}$ \\
\hline Number of rounds & 1000 \\
\hline
\end{tabular}

\subsection{DISCUSSION}

The simulation results in Figure 8 showed that the MQTT protocol consumes more than $20 \%$ of the energy than the MQTT-SN protocol.

Second, the simulation results (Figure 9) showed that incorporating MQTT-SN QoS levels into the data delivery process on our WSN-based 2SIEC system can reduce a percentage of MQTT-SN broker messages for data retransmission in the event of a gateway or controller failure, and will subsequently reduce the power consumption of the sensor nodes and increase the life of the WSN.

QoS1 consumes less power than the other two levels QoS0 and QoS2, QoS2 consumes more power than the other two levels QoS1 and QoS0, QoS0 consumes 1.23\% more power than QoS1, QoS2 consumes 6.63\% more power than QoS1, QoS0 consumes $5.17 \%$ less power than QoS2.

Based on these results, it can be said that if there is quality control in data delivery and it is possible to reorganize the sensor topology in a network, this would contribute to increasing the lifetime of the wireless sensor network (Figure $9)$.

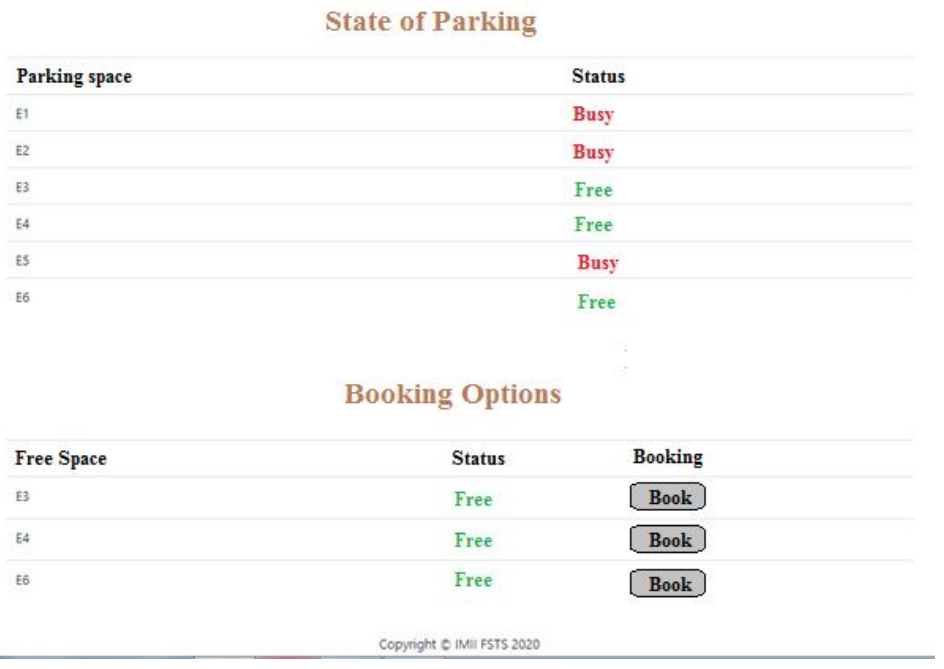

Figure 7:Interactive web page, by drivers 


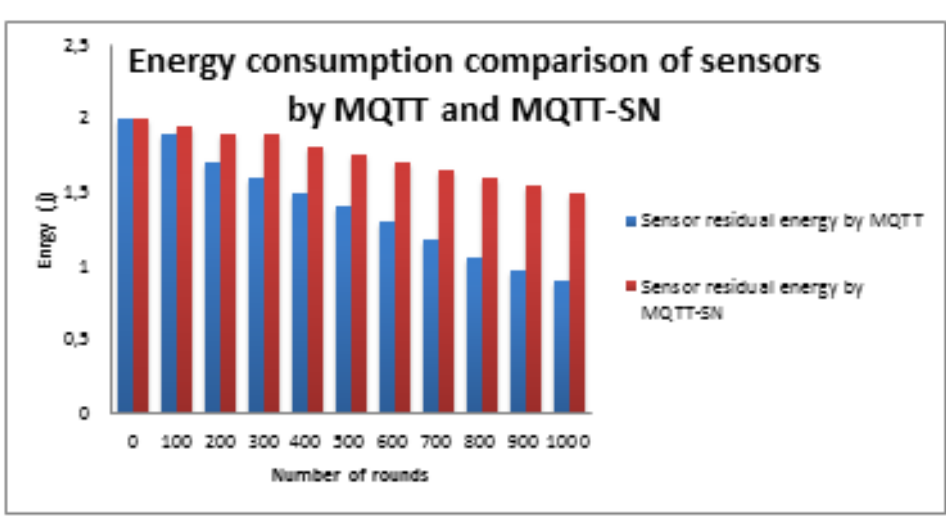

Figure 8.Comparison of the energy consumption of our WSN by MQTT and MQTT-SN

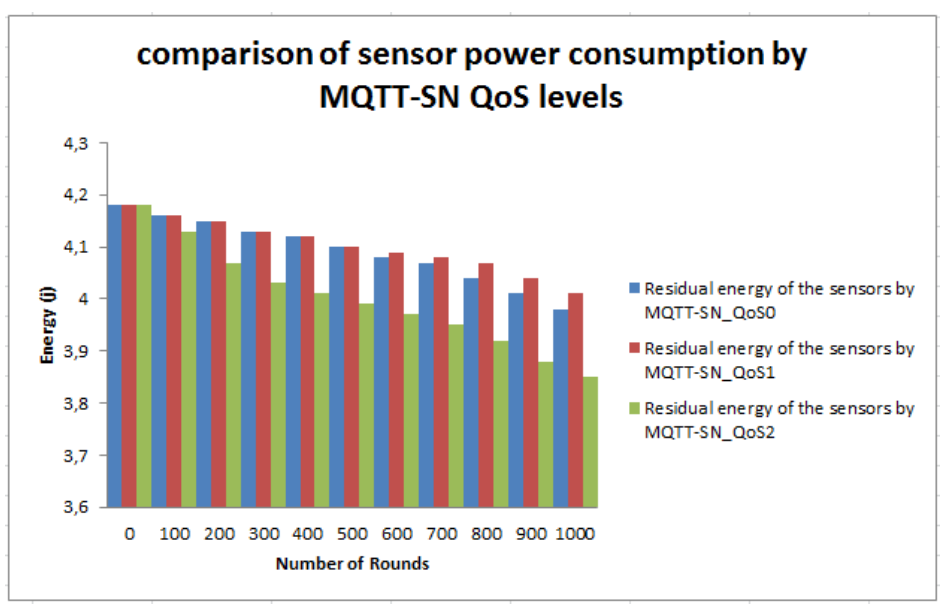

Figure 9.Comparison of our WSN power consumption by MQTT-SN QoS levels

\section{CONCLUSION}

The industrial world has undergone several revolutions that have changed the daily lives of citizens, and more particularly the modes of transport of goods and people. Man has been able to develop means of transport (cars, lorries, buses, etc.), the number of which is constantly increasing and which have far exceeded the capacity of the infrastructure on offer, leading to congestion, particularly in car parks. Thus, in order to contribute to decongestion in car parks and to make life easier for drivers, this study, entitled 2SIEC, aims to find a solution to this kind of problem. It consists in making parking intelligent by allowing access and management of free spaces via a web platform or an Android application. We have also shown in this study that the MQTT-SN protocol in its QoS1 quality of service is more efficient in the energy optimization of our WSN used in our 2SIEC system.

\section{ACKNOWLEDGEMENT}

The authors are very much thankful to the unanimous reviewers of the paper and editors of the journal for their constructive and helpful comments that improved the quality of the paper.

\section{REFERENCES}

1. E. S. Pramukantoroet H. Anwari, « An Event-based Middleware for Syntactical Interoperability in Internet of Things », IJECE, vol. 8, no 5, p. 3784, oct. 2018, doi: 10.11591/ijece.v8i5.pp3784-3792.

2. H. Ouldzira, H. Lagraini, A. Mouhsen, M. Chhiba, et A. Tabyaoui, «MG-leach: an enhanced leach protocol for wireless sensor network », IJECE, vol. 9, no 4, p. 3139, août 2019, doi: 10.11591/ijece.v9i4.pp3139-3145.

3. Sivapriya, J., Akaveeti, J., Kotha, K., \&Sushmitha, M. (2018). Enhanced Smart Parking System using Sensor Technology. International Journal of Emerging Technologies in Engineering Research (IJETER), 6(10).Mainetti, L., Palano, L., Patrono, L., Stefanizzi, M. L., \&Vergallo, R. (n.d.). Integration of RFID and WSN Technologies in a Smart Parking System.

4. O. Hicham, A. Mouhsen, H. Lagraini, A. Tabyaoui, et M. Chhiba, « Smart monitoring information system based on RF $433 \mathrm{Mhz}$ (SMIS) », IJECE, vol. 9, no 6, p. 5143, déc. 2019, doi: 10.11591/ijece.v9i6.pp5143-5149. https://doi.org/10.11591/ijece.v9i6.pp5143-5149

5. YanxuZheng, S. Rajasegarar, et C. Leckie, « Parking availability prediction for sensor-enabled car parks in smart cities », in 2015 IEEE Tenth International Conference on Intelligent Sensors, Sensor Networks and Information Processing (ISSNIP), Singapore, avr. 2015, p. 1-6, doi: 10.1109/ISSNIP.2015.7106902.

6. I. G. B. B. Nugraha et F. R. Tanamas, «Off-street parking space allocation and reservation system using event-driven algorithm », in 2017 6th International Conference on Electrical Engineering and Informatics (ICEEI), Langkawi, nov. 2017, p. 1 - 5, doi: 10.1109/ICEEI.2017.8312456.

7. A. Sharma, R. Chaki, et U. Bhattacharya, « Applications of wireless sensor network in Intelligent Traffic System: A review », in 2011 3rd International Conference on Electronics Computer Technology, Kanyakumari, India, avr. 2011, p. 53 - 57, doi: 10.1109/ICECTECH.2011.5941955.

8. Miao Wu, Ting-Jie Lu, Fei-Yang Ling, Jing Sun, et Hui-Ying Du, « Research on the architecture of Internet of Things », in 2010 3rd International Conference on Advanced Computer Theory and Engineering(ICACTE), Chengdu, China, août 2010, p. V5-484-V5-487, doi: 10.1109/ICACTE.2010.5579493.

9. Angeline, R., \&Eshasree, M. (2018). Intelligent Transportation System Using IOT. International Journal of Emerging Technologies in Engineering Research (IJETER), 6(11). Retrieved from http://www.gisdevelopment.net/application/utility/transp ort/mi03164pf.htm. 
10. M. Karaliopoulos, K. Katsikopoulos, et L. Lambrinos, « Bounded rationality can increase parking search efficiency », in Proceedings of the 15th ACM international symposium on Mobile ad hoc networking and computing - MobiHoc '14, Philadelphia, Pennsylvania, USA, 2014, p. 195 - 204, doi: $10.1145 / 2632951.2632955$.

11. E. Kokolaki, M. Karaliopoulos, et I. Stavrakakis, « Leveraging Information in Parking Assistance Systems », IEEE Trans. Veh. Technol., vol. 62, no 9, p. 4309-4317, nov. 2013, doi: 10.1109/TVT.2013.2269015.

12. T. Rajabiounet P. Ioannou, " On-Street and Off-Street Parking Availability Prediction Using Multivariate Spatiotemporal Models », IEEE Trans. Intell. Transport. Syst., vol. 16, no 5, p. 2913 -2924, oct. 2015, doi: 10.1109/TITS.2015.2428705.

13. S. Pullola, P. K. Atrey, et A. El Saddik, « Towards an Intelligent GPS-Based Vehicle Navigation System for Finding Street Parking Lots », in 2007 IEEE International Conference on Signal Processing and Communications, Dubai, United Arab Emirates, 2007, p. 1251-1254, doi: 10.1109/ICSPC.2007.4728553.

14. Faheem, S. A. Mahmud, G. M. Khan, M. Rahman, et H. Zafar, "A Survey of Intelligent Car Parking System », Journal of Applied Research and Technology, vol. 11, no 5 , p. 714 - 726, oct. 2013, doi: 10.1016/S1665-6423(13)71580-3.

15. R. Arnottet J. Rowse, "Curbside parking time limits », Transportation Research Part A: Policy and Practice, vol. 55, p. 89-110, sept. 2013, doi: 10.1016/j.tra.2013.07.009.

16. Hae Don Chon, D. Agrawal, et A. El Abbadi, « NAPA : Nearest Available Parking lot Application », in Proceedings 18th International Conference on Data Engineering, San Jose, CA, USA, 2002, p. 496-497, doi: 10.1109/ICDE.2002.994767. 